\title{
Kansei Modeling for Delight Design based on 1DCAE Concept
}

\author{
Koichi Ohtomi \\ The University of Tokyo, JAPAN, koichi.ohtomi@delight.t.u-tokyo.ac.jp
}

\begin{abstract}
The main task of product development is to develop a good product at lower cost and to bring it to market in a shorter period. Conventional computer-aided design and computer-aided engineering $(\mathrm{CAD} / \mathrm{CAE})$ systems are well established in this regard. However, although upstream design is particularly important in product development to add value and incorporate the required functions, it is difficult to apply conventional shapebased $\mathrm{CAD} / \mathrm{CAE}$ systems to the upstream design stage due to the lack of design information at that stage. As a solution to this issue, we are proposing the development of a design framework called "1DCAE", which can be applied to the early design stage of product development including the conceptual and functional design phases. The 1DCAE concept can be applied not only better design, must design, but delight design. Here we introduce how 1DCAE concept applies to delight design and its core technology of kansei modeling.
\end{abstract}

Keywords: 1DCAE, delight design, kansei, modeling, better design, must design, CAD/CAE

\section{Introduction}

The 1DCAE based on a simple but an essential model is a methodology, a method and a tool to support the whole design from the early design stage to the detail design stage and to glance from mechanical, electrical to software design. 1D means to capture the essence of things including physical phenomena and to express by simple model to be easy to understand. 1DCAE can realize the evaluation by CAE from the upstream to downstream design. There are three kinds of design fields to apply 1DCAE concept. They are better design to realize the low cost and better performance, must design to realize the safety and security, and delight design to get to customers' heart. Delight design has become more important than ever in manufacturing with the diversification of recent customer requirement. On the other hand, we need to model the characteristics of kansei in delight design in addition to the conventional physical model for better and must design. Kansei is originally a Japanese word that refers to the sensitivity of a human sensory organ at which sensations or perceptions take place in response to stimuli (e.g., a product) from the external world. Kansei includes evoked senses, feelings, emotions, and impressions. The word kansei has begun to be used internationally because there is no suitable translation in English. Figure 1 shows the image of kansei.

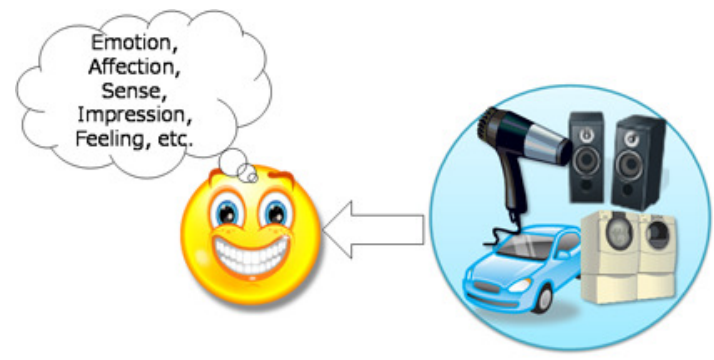

Figure 1: what is kansei?

Here we call the model to express the characteristics of kansei "the kansei model". Kansei modeling based on 1DCAE can introduce kansei into the flow of manufacturing and provide a platform of delight design that does not depend on intuition and experience. Kansei model is implemented on the 1D tool based on Modelica language. Kansei model enables quantitative evaluation because it starts from the physical model.

\section{Concept of 1DCAE}

Product development starts in a conceptual design and progresses with functional design, a layout design, a structural design, and a manufacturing design. However, in the early design stage of design process design information is ambiguous. Therefore, it is difficult in the early design stage of a design to apply conventional design tool such as $\mathrm{CAD} / \mathrm{CAE}$. In a second half of design process $\mathrm{CAD} / \mathrm{CAE}$ will become applicable, but in this stage, there are many design restrictions and then flexibility of design decreases. Moreover, not only $\mathrm{CAD} / \mathrm{CAE}$ but rapid prototyping (RP) and experiment are possible in the second half of design process. Therefore, the design methodology/method to be applicable from the early stage of design process is desired.

In the matured industrial field, it is sufficient for a design to start from a layout design and a structural design, but in the industrial field that is strongly depends on the voice of customer ( $\mathrm{VoC})$, it is required to start from the upper stage of design process to get the VoC before starting a detailed design.

Then, we propose the 1DCAE concept to be applicable from the early stage of design process. "1D" doesn't 
mean one dimensional, but it means simple but essential expression of phenomena. By applying 1DCAE, it becomes to be able to evaluate from the upper design stage to the lower design stage by CAE. The CAE mentioned here means not a simulation but the original concept of Computer-Aided Engineering.

In case of a product design by 1DCAE, the concept of product and/or system should be expressed in functional basis and then can be analyzed by system simulation tool based on Modelica before creating a shape. This process enables the totally appropriate design in the early stage of design process. The output of 1DCAE is the input of CAD/CAE. Figure 2 shows the design process based on the 1DCAE concept.

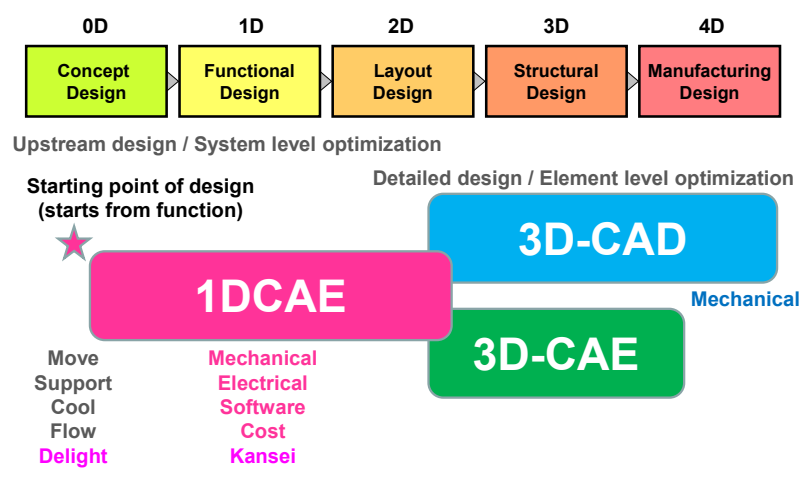

Figure 2: Design process based on 1DCAE concept

The relation between 1DCAE and 3D-CAE is shown in Figure 3. In 1DCAE, the target of a product development is set up and a conceptual design and a functional design are performed. By considering the function of a product, the preliminary decision of the design specification is carried out, and it delivers to 3DCAE. In 3D-CAE, a structural design and a layout design are performed based on the specification received from 1DCAE. 3D-CAE is a part to consider structure, and a conventional CAD/CAE demonstrates their power. The result of $3 \mathrm{D}-\mathrm{CAE}$ is fed back to 1DCAE and performs functional verification as a system. The 1DCAE in a broad sense is a design framework combination of $1 \mathrm{DCAE}$ and $3 \mathrm{D}-\mathrm{CAE}$.

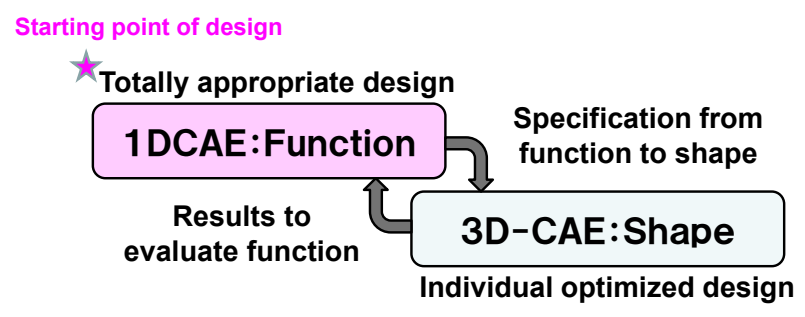

Figure 3: Relation between 1DCAE and 3D-CAE

\section{1DCAE and delight design}

The 1DCAE has a different configuration depending on the purpose of design. We classify into three kinds of design depending on the purpose. Three kinds of design are classified according to Kano model as shown in Figure 4.

I. Must design equivalent to commonplace quality:

Design for assurance design. Many of the trouble caused by neglecting this design. It is not so easy to evaluate the designers' effort for this design, but the basics of design. II. Better design equivalent to performance:

Because the target is so clear, it is easy to approach for this design. On the other hand, it will fall into cost competition eventually. The purpose of this design is to cost minimization, time to market (TTM) minimization, and performance maximization.

III. Delight design equivalent to attractive quality: Design concept is the most important in this design. Many of hit products come from this area.

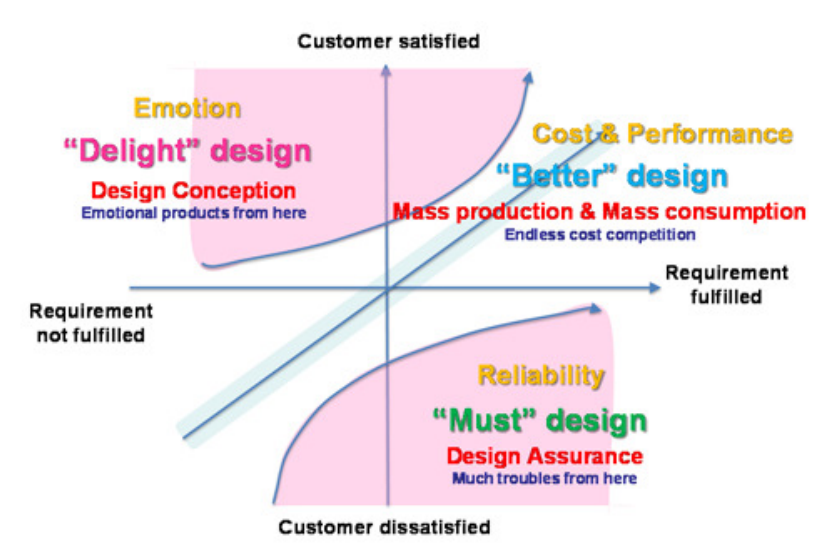

Figure 4: Three kinds of design

It is crucial to produce emotional products (delight design) in addition to must design and better design in the future industry. Therefore we focus on the delight design in this paper. Figure 5 shows an example of 1DCAE delight design to apply home appliance. Conventionally, product sound design focused on reducing the noise level. Here we propose another aspect of product sound design to add values to products. To capture the sound as a value rather than noise, it requires the extraction of the potential needs of customers on the sound. Because customer needs are diverse, it is important to set the target in consideration of this effect. On the other hand, there exists a design metrics of noise level in case of noise reduction. When we treat the sound as a value, we need to introduce new metrics to express comfortable sound as designs can understand. 1DCAE delight design to product sound design is to the process to extract customer needs, develop the metrics of sound, set the target sound, and realize as products. Product design performed based on the requirement derived from 1DCAE process, sound 
data from prototyping mapped on the metrics of sound to validate the performance, and finally we got the new product to satisfy the target sound with comfortableness. Because delight design was conducted from the early stages of product development together with must design and better design, there was no additional cost and no negative impact on performance.

Delight design treats kansei. Kansei is also one of the functions of products, but it is difficult to combine function and structure directly. Therefore, we combine psychological domain function (worth) with structure via physical domain function of sound quality metrics as shown in Figure 6. Sound quality metrics which was created as a result of the field of psychoacoustic, and can represent the perception about the human sensitivity to sound as objective numerical value that was analyzed as sound data. As shown in Figure 6, loudness, sharpness, roughness, and fluctuation strength are often used as a representative sound quality metrics. These metrics enable us to express comfortable sound as a common language. In addition, sound quality metrics will be kansei metrics for sound, which will be described later.

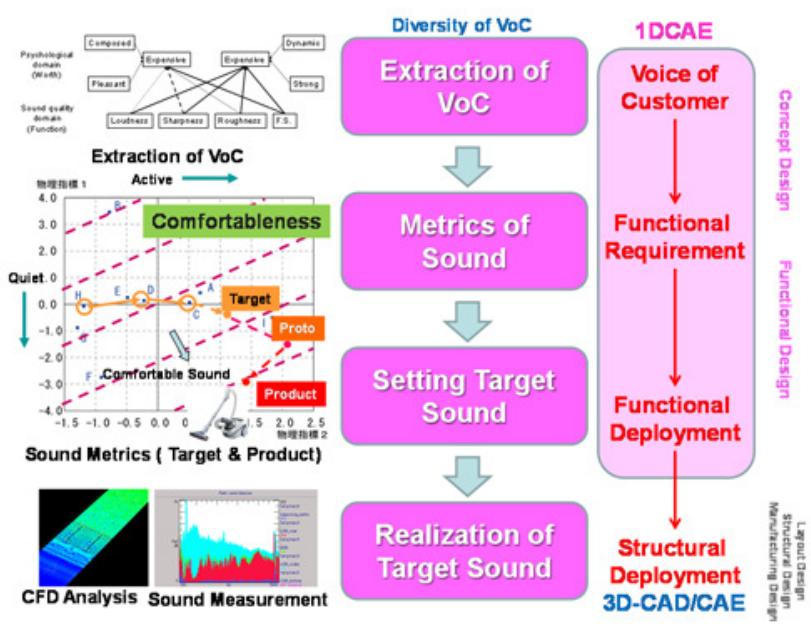

Figure 5: Example of delight design based on 1DCAE



Figure 6: Combination of psychological domain with physical domain via sound quality domain

\section{Kansei modeling based on 1DCAE}

We introduce the kansei modeling based on 1DCAE concept to realize delight design. The technology to capture the kansei is defined as the kansei modeling and the resulting model as the kansei model. 1DCAE is done by using the so-called 1D tool based on Modelica language. Figure 7 shows a model image of the hair dryer in which the left half is for the physical model and the right half for the kansei model. Starting from the physical model, then kansei model is built by cooperation with the kansei database. In case of dryer, air flow, sound, and handling are related to kansei.

Designers perform the delight design by using 1D tool with kansei model. They can check the degree of attainment for their ideas by calculating the attractive metric on 1D tool. Results of delight design obtained in this process is sent to the mechanical design and circuit design processes to perform the tangible design.

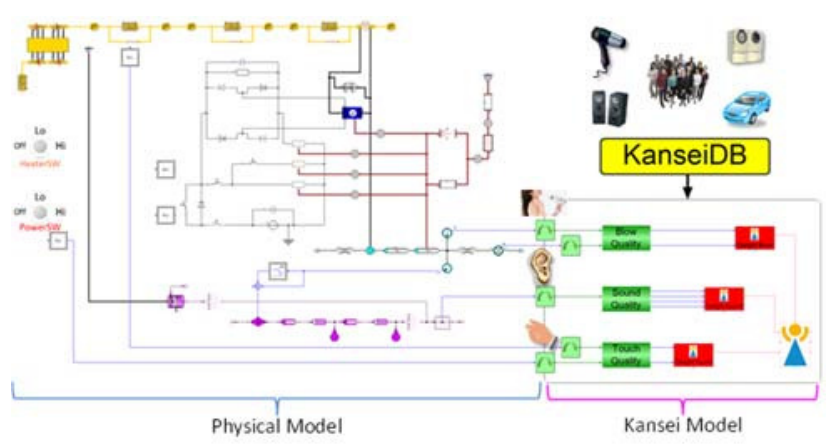

Figure 7: Image of kansei modeling

\section{Kansei modeling in case of sound design}

Then, we will introduce the kansei modeling in case of sound design. The physical model is derived by analyzing the existing product as truly as possible considering the physical law. Figure 8 shows the 1D physical model of a dryer. The basic elements such as a fan, a motor, and a heater and their related structure, mechanism, housing, electronics, and software are expressed by using the 1D tool software.

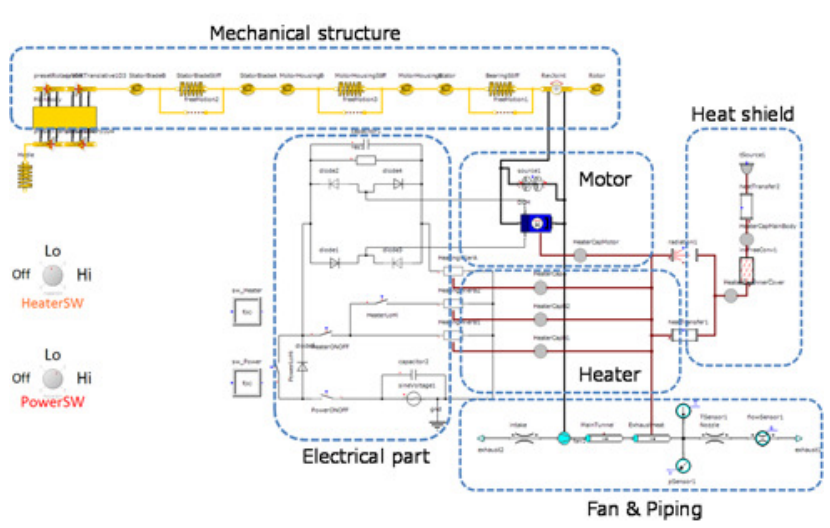

Figure 8: 1D physical model 
Figure 9 shows the FS (function and Structure) map in order to set the development goals of a dryer. The FS map informs us the design items related to must, better, and delight design. From the extracted delight design items, we selected the structure to create comfortable sound.

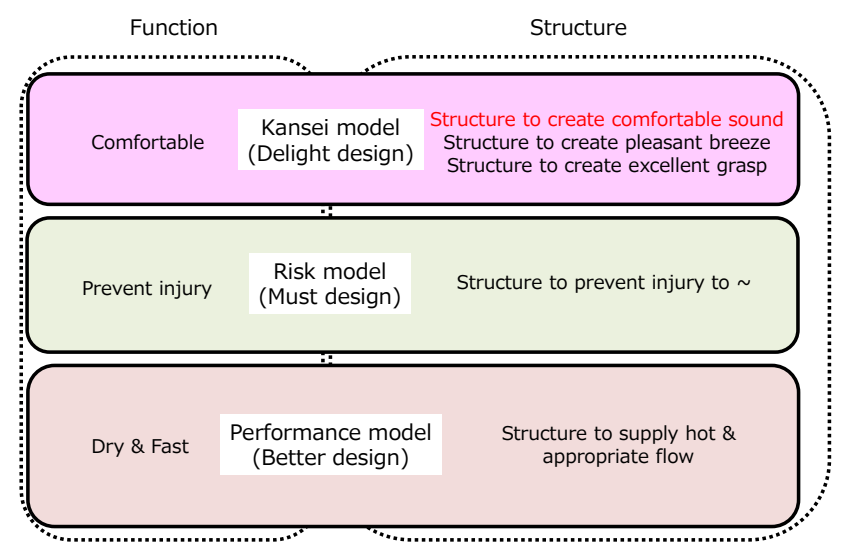

Figure 9: FS (Function Structure) map

The acoustic model should be added to the $1 \mathrm{D}$ physical model as shown in Fig.8 as the first step of the kansei model for sound. Figure 10 shows the 1D physical model with acoustics.

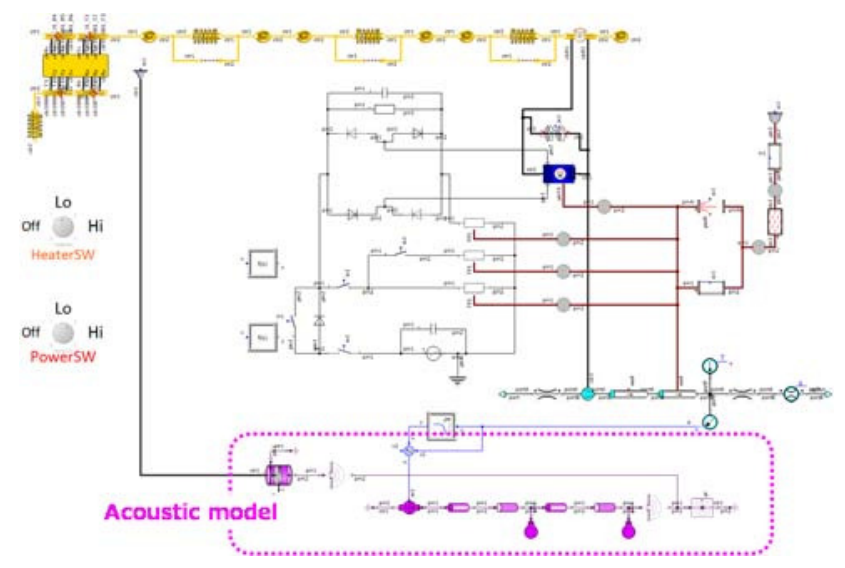

Figure 10: 1D physical model with acoustic model

Then we build the kansei model for sound as shown in Figure 11 according to the same procedure as shown in Figures 5 and 6.

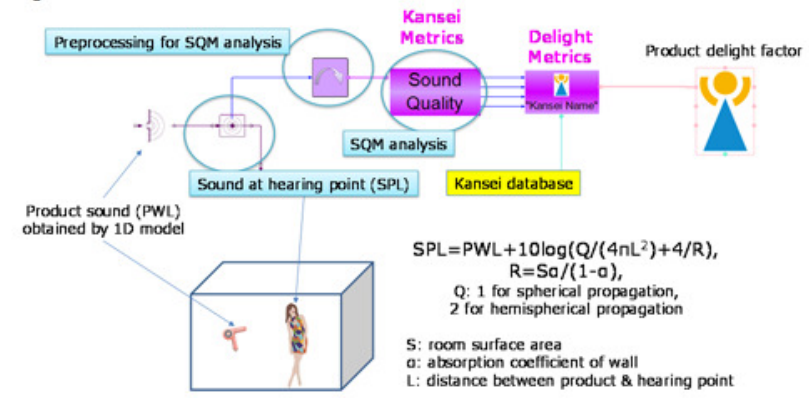

Figure 11: 1D kansei model for sound
Figure 12 shows the example of simulation results to describe the sound quality metrics and FFT when a dryer starts to operate from power off condition to low mode and then high mode.

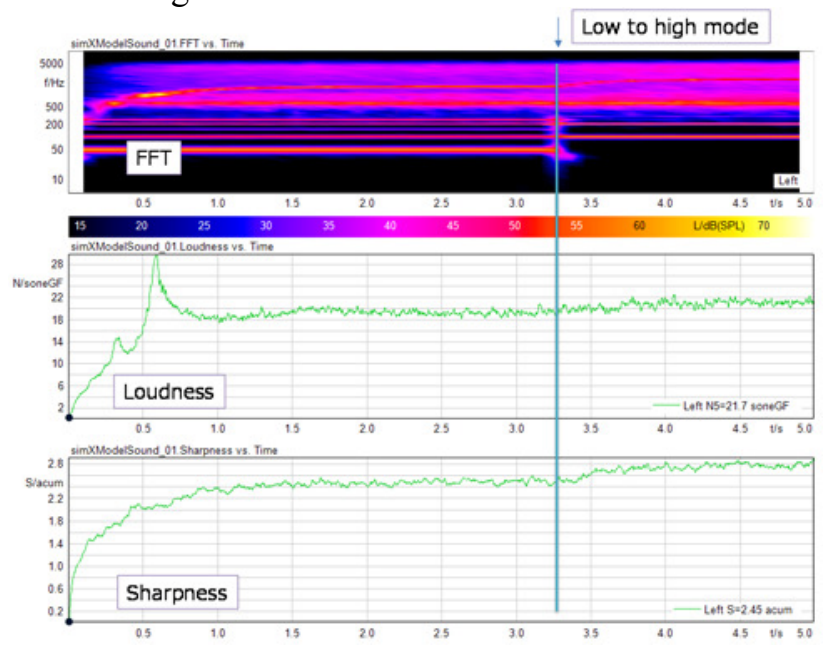

Figure 12: Example of simulation results

\section{Future work}

We aim at the delight design for kansei in general. In the future, kansei models not only for sound quality but for visual quality, flow quality, and touch quality will be developed as shown in Figure 13.

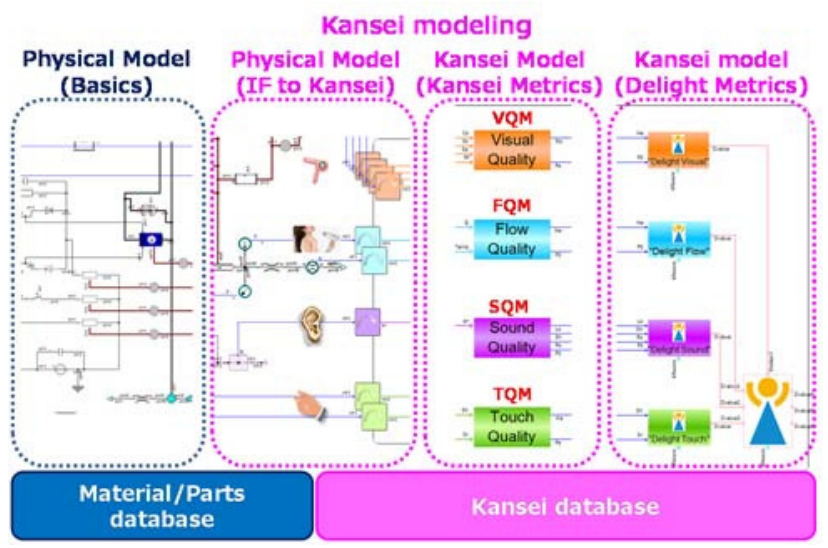

Figure 13: Total image of 1D kansei model

\section{Conclusion}

We introduced the kansei modeling based on 1DCAE concept. Kansei model was implemented on the 1D tool based on Modelica language in case of sound design. Kansei model enabled quantitative evaluation because it started from the physical model.

\section{Acknowledgements}

This research was supported by New Energy and Industrial Technology Development Organization (NEDO) of Japan, and we would like to thank them for their assistance. 


\section{References}

Ohtomi, K. and Hato, T., "Design Innovation Applying 1DCAE", July, TOSHIBA REVIEW. (2012)

Ohtomi, K., Design of Worth for Customer Product Development, What is "What's the Design"? Special Issue of Japanese Society for the Science of Design vol.16-2 no.62, 31-38, 2009

Ohtomi, K., Hosaka, R., 2008, "Design for product sound quality", Internoise2008.

Ohtomi, K. and Hosaka, R., "Product Sound Design", September, TOSHIBA REVIEW. (2007)

Ohtomi, K., "Importance of Upstream Design in Product Development and Its Methodologies", January, TOSHIBA REVIEW. (2005)

Yanagisawa, H., Murakami, T., Noguchi, S., Ohtomi, K., Hosaka, R., 2007, "Quantification method of diverse kansei quality for emotional design application of product sound design”, ASME DETC2007-34627.

Zwicker., E, 2006, "Psychoacoustics: Facts and Models", Springer, 3rd Edition, Springer-Verlag, New York Inc. 